\title{
The immuno-mass spectrometry chemical microscope
}

\author{
Dominic J. Hare ${ }^{1,2}$, Olga Shimoni ${ }^{3}$ and David P. Bishop ${ }^{2 *}$
}

${ }^{1}$ School of BioSciences, The University of Melbourne, BioSciences 1, Tin Alley, Parkville, Victoria 3010, Australia.

${ }^{2}$ Atomic Medicine Initiative, Faculty of Science, University of Technology Sydney, P.O. Box 123, 15 Broadway, Ultimo, New South Wales 2007, Australia

${ }^{3}$ Institute for Biomedical Materials and Devices, Faculty of Science, University of Technology Sydney, P.O. Box 123, 15 Broadway, Ultimo, New South Wales 2007, Australia

*Correspondence: david.bishop@uts.edu.au (D.P. Bishop)

Twitter: @DominicJHare (DJH); @OIgainAus (OS) @dp_bish (DPB)

\begin{abstract}
Elemental mass-spectrometry imaging has evolved beyond mapping biometals in tissue sections. The continually improving sensitivity and spatial resolution of chemical imaging technology has the potential to revolutionize immunohistochemistry. We explore how simple modifications to routine immunostaining protocols that integrate 'immuno-mass spectrometry imaging' are making in-situ quantitative protein mapping a reality.
\end{abstract}

Keywords: Imaging mass spectrometry; immunohistochemistry; LA-ICP-MS; quantitative bioimaging. 


\section{'Antibodies are big business'}

The global antibody business is worth $\$ 80$ billion, around $95 \%$ of which is the clinical diagnostics and immunotherapeutics industries. The remaining $\$ 3$ billion, accounting for around a third of all the reagents a bioscience laboratory purchases each year [1], is spent on antibodies for research purposes.

\section{Immunoassays: measures and markers}

Immunoassays (Box 1) are routine bioanalytical techniques essential to life science and clinical research laboratories that use antibody-antigen interactions to detect, quantify, and visualize biomolecules. Western blots and enzyme-linked immunosorbent assays (ELISAs) can accurately quantify numerous biomolecules, from total hemoglobin levels in the blood to low abundance proteins encoded by genes with single point mutations. Immunohistochemistry (IHC, Box 1) applies the same principles to tissue sections and cells for assessing the spatial distribution of an antigen. As versatile and specific as immunoassays are, no single technique can simultaneously evaluate how much of what biomolecule is where.

\section{What, where, and how much?}

Considering the sheer number of proteins, peptides, and post-translational modifications involved in any pathological process, the development of quantitative IHC methods has the potential to transform understanding of the molecular basis of diseases. Identifying and monitoring changes in levels of biomolecules involved in cell signaling, mitochondrial function, and cell death mechanisms can be used for developing new therapeutic targets and predictive biomarkers.

\section{Shortfalls of the immunohistochemical 'stain'}

Interpretation of IHC is inherently subjective. The quality of reagents used and observer bias are two primary sources of variance, and pathological assessment is made based on the intensity of a color reaction in comparison to a positive control [2]. In the clinic, IHC is typically used to visualize the spread of a specific diagnostic marker and staining is used to assign biopsies to consensus classes, such as tumor grading. 'Relative staining intensity' can be used as a proxy for the amount of antigen present within experiments, though the considerable number of inter-operator variables makes IHC entirely qualitative as an indicator of biomolecule levels within defined microscopic areas. Only a handful of IHC methods have 
been approved by regulatory bodies for commercial and clinical use, all of which are qualitative with respect to regional antigen levels. In a 2014 systematic review by the College of American Pathologists, only $5.8 \%$ of examined IHC protocols were deemed to be of sufficient quality for further analysis as supporting evidence for analytical validation guidelines. A lack of standardization and consistency across laboratories, and inadequate testing to ensure assay validity were identified as the exclusionary factors [3]. Combining the principles of analytical validation with advanced imaging technology can help overcome these shortcomings and introduce new capabilities for researchers and clinicians.

\section{iMSI: a quantitative complement to immunohistochemistry}

Immuno-mass spectrometry imaging (iMSI) uses laser ablation (Box 1) and inductively coupled plasma-mass spectrometry (LA-ICP-MS, Box 1 and Figure 1A) to complement standard protocols and overcome sensitivity and quantitative limitations of IHC. Chromogenic and fluorescent reporters are replaced with metals as pre-absorbed nanoparticles or ions affixed via chemical linkers. The ideal proxies for fluorescent reporters in iMSI are high-mass exogenous elements typically not present in biological tissue, such as rare earth elements (REEs). The first example of $\mathrm{iMSI}$ in 2005 used both $\mathrm{Ni}^{2+}$ (an endogenous element that reacts with the 3,3'-diaminobenzidine chromogen to produce a brown stain) and Eu ${ }^{3+}$-conjugated secondary antibodies to visualize Alzheimer's-like plaques in brains of transgenic mice overexpressing mutated $\beta$-amyloid protein associated with early-onset disease [4].

Increasing the number of REEs adherent to a single antibody increases sensitivity. Metalchelating polymers (MCPs, Box 1) that bind multiple REE ions per linker, and conjugation of upconversion nanoparticles (UCNPs, Box 1) with a lanthanide-containing inorganic matrix increases the number of REE atoms per antibody by up to several orders of magnitude. Luminescent properties of UCNPs [5] are also ideal for fluorescent microscopy prior to iMSI analysis. The images produced are calibrated against known amounts of UCNPs embedded in matrix-matched standards for external quantification of total REE levels (Figure 1B).

As with any emerging analytical method, advances in LA-ICP-MS technology have driven further development to a point where iMSI has developed distinct advantages over traditional IHC as a research tool with clear potential for clinical translation, provided the rigid protocols of analytical method validation are appropriately applied.

\section{...with new opportunities...}


Multiplexing imaging (Box 1) examines the spatial or quantitative relationship between multiple antigens in a single sample. This approach traditionally uses several secondary antibodies with preabsorbed fluorophores; however, overlap of emission spectra and cross-species reactivity of antibodies limits the number of simultaneously-detectable analytes and requires specialized microscopes with appropriate excitation sources and filters. A substantial advance was made by Giesen and colleagues [6] when demonstrating how LA-ICP-MS imaging could be used to visualize 32 antigens simultaneously (Figure 1C). Affixing MCPs and REEs to primary antibodies eliminates the restrictions presented by cross-species reactivity when using secondary antibodies with bound chemical reporters for signal amplification.

The system used by Giesen and colleagues was termed imaging mass cytometry (Box 1) and is now commercially available. Although an incredibly powerful technique for mapping biomolecular pathways involved in complex diseases such as breast cancer, imaging mass cytometry is not trivial to use. Pre-labeled kits are available to purchase for outlining specific biochemical pathways, but custom-designed experiments require isotopically enriched lanthanides and proprietary reagents, including the MCP, to individually prepare each primary antibody. The degree of labeling differs per preparative reaction, limiting potential quantitative applications, and is a time consuming process.

Industry-standard LA-ICP-MS designs are capable of reproducing several aspects of imaging mass cytometry, with key advantages and several limitations, depending on the approach used. As a quantitative tool, conjugated nanoparticles in place of MCPs dramatically improves sensitivity by increasing the number of detectable atoms in line with nanoparticle diameter (Figure 1E). Accurate characterization of nanoparticle dimensions using electron microscopy and REE content by ICP-MS should be used to determine the ideal nanoparticle reporter what gives adequate sensitivity while also limiting potential steric hindrance that reduces total antigen-antibody binding. Chromatography with ICP-MS detection and quantification by isotope dilution can determine the number of metal atoms bound to a single antibody using the sulfur content of the immunoglobulin as an analytical proxy [7].

\section{...and new challenges}

Laser ablation technology is the area undergoing the most rapid development to support iMSI applications. Stable and reliable laser sources are needed for extended analysis times, and sub-cellular spatial resolution requires ablating micron-scale areas in sample chambers able to efficiently transport ejected material to the ICP-MS in $<10 \mathrm{~ms}$ when scanning at high speed. Image post-processing can also improve spatial resolution that is quickly approaching the 
nanoscale using data reconstruction based on the principles of super-resolution microscopy, where orthogonal scans are taken at a low resolution and are combined into a single highresolution image [8].

Quadrupole and time-of-flight (TOF) mass analyzer configurations are most commonly used for iMSI, both with advantages and limitations. Quadrupoles dominate the market, and routinely outperform TOF analyzers with respect to sensitivity, though the sequential ion scanning nature of quadrupole design limits the number of analytes and introduces a degree of skewing (Box 1) in the resulting image. This can be somewhat mitigated by matching the laser pulse rate to the ion acquisition times [9], though only simultaneous sampling of the plasma and detection using a TOF mass analyzer can fully negate this imaging artifact. Bodenmiller and colleagues' work using the TOF-based imaging mass cytometer has provided unprecedented insight into disease-specific pathways [10], though the design optimized for detecting higher mass elements is unavble to monitor levels of relevant endogenous metals with a mass $<80$ amu.

\section{Where to now?}

Quantitative iMSI is not just a research tool. Ideally, iMSI should improve on IHC and develop companion diagnostics that are accepted by regulatory authorities. Sample preparation steps must be consistent with routine IHC protocols and easily integrated into laboratory workflows. Most importantly, like any new technique, it must undergo extensive validation according to both the principles of analytical method development, while also adhering to the guidelines set by the College of American Pathologists [3]. The quality of the data produced is dependent on the antibody used; there are currently over 4.5 million antibodies listed in the CiteAb, up from 2 million in 2015 [11]. It is crucial that benchmarking protocols using known positive and negative controls be developed for iMSI (Figure 1D) [12].

Standardization must also extend to preparation methods to accelerate uptake of iMSI and to produce translatable results. These standards must be applicable across multiple imaging platforms to enable direct comparisons between laboratories independent of reagents and instrumentation [12]. Relative quantification between samples is straightforward, and absolute quantification is possible with knowledge of the number of metal atoms per antibody, and extensive characterization of antibody-antigen stoichiometry should be a major research focus.

\section{Concluding remarks}


Quantitative iMSI has the potential to redefine biomarker discovery by introducing cellular localization, absolute amounts, and networks of biomolecular interactions as new targets. While technological advances are primarily driven by industry, now is the time for the field to expedite the development of standardized protocols and image acquisition and analysis methods independent of the instrumentation for international round-robin validation studies of iMSI as a companion to traditional diagnostics.

\section{Acknowledgements}

DJH is supported by a research fellowship from the National Health and Medical Research Council (GNT1122981). OS is supported by the Australian Research Council Industrial Training Center (IH150100028); and acknowledges the Australian Research Council and National Health and Medical Research Council for financial support (APP1101258). DPB is supported by an Australian Research Council Discovery Early Career Researcher Award (DE180100194) and a USA National Institute of Health R21 Exploratory/Development Grant (1R21AR072950-01A1).

\section{Conflict of interest statement}

DJH receives infrastructure, applications, and research support from Agilent Technologies through the National Health and Medical Research Council Career Development Fellowship (Industry) scheme. He also receives infrastructure and applications support from Elemental Scientific Lasers LLC. 


\section{References}

1. 2017 Biocompare Antibody Report: Market Overview and Industry Survey, CompareNetworks, Inc., San Francisco, 2017.

2. Taylor, C.R. (2014) Predictive Biomarkers and Companion Diagnostics. The Future of Immunohistochemistry: "In Situ Proteomics," or Just a "Stain"? Applied Immunohistochemistry and Molecular Morphology 22 (8), 555-561.

3. Fitzgibbons, P.L. et al. (2014) Principles of Analytic Validation of Immunohistochemical Assays: Guideline From the College of American Pathologists Pathology and Laboratory Quality Center. Archives of Pathology and Laboratory Medicine 138 (11), 1432-1433.

4. Hutchinson, R.W. et al. (2005) Imaging and spatial distribution of $\beta$-amyloid peptide and metal ions in Alzheimer's plaques by laser ablation-inductively coupled plasma-mass spectrometry. Analytical Biochemistry 346 (2), 225-233.

5. Chen, Y. et al. (2020) Dispersion stability and biocompatibility of four ligand-exchanged NaYF4: Yb, Er upconversion nanoparticles. Acta Biomaterialia 102, 384-393.

6. Giesen, C. et al. (2014) Highly multiplexed imaging of tumor tissues with subcellular resolution by mass cytometry. Nature Methods 11 (4), 417-422.

7. Clases, D. et al. (2019) SEC-ICP-MS and on-line isotope dilution analysis for characterisation and quantification of immunochemical assays. Analytical and Bioanalytical Chemistry 411 (16), 3553-3560.

8. Westerhausen, M. et al. (2019) Super-resolution reconstruction for two and threedimensional LA-ICP-MS bio-imaging. Analytical Chemistry 91 (23), 14879-14886.

9. Van Malderen, S.J.M. et al. (2018) Considerations on data acquisition in laser ablationinductively coupled plasma-mass spectrometry with low-dispersion interfaces.

Spectrochimica Acta Part B Atomic Spectroscopy 140, 29-34.

10. Schapiro, D. et al. (2017) histoCAT: analysis of cell phenotypes and interactions in multiplex image cytometry data. Nature Methods 14 (9), 873-876.

11. Bradbury, A. and Plückthun, A. (2015) Reproducibility: Standardize antibodies used in research. Nature 518 (7537), 27.

12. Bodenmiller, B. (2016) Multiplexed Epitope-Based Tissue Imaging for Discovery and Healthcare Applications. Cell Systems 2 (4), 225-238.

13. Zhu, T. et al. (2020) Metallo-Polyelectrolytes: Correlating Macromolecular Architectures with Properties and Applications. Trends in Chemistry 2 (3), 227-240.

14. Chen, B. and Wang, F. (2020) Emerging Frontiers of Upconversion Nanoparticles. Trends in Chemistry. 


\section{Box 1 - A lexicon of key concepts and terminologies for iMSI}

Immuno-mass spectrometry imaging (iMSI): antibodies are conjugated to rare and exotic metals, such as lanthanides. Stained sections are then imaged using LA-ICP-MS, using antibody-metal conjugates as proxies for target antigens. A number of quantitative methods can then be used to estimate total metal levels, and thereby amount of antibody-antigen binding.

Immunoassay: antibodies raised to recognize specific structural and chemical properties of biomolecules called epitopes are used to bind to antigens containing these regions. Chemical reporters, normally fluorophores, attached to either the primary antibody or an additional secondary antibody for signal amplification are used to detect antibody-antigen complexes. Quantitative methods include Western blotting and enzyme-linked immunosorbent assays (ELISA), and in situ detection uses immunohistochemistry (see below).

Immunohistochemistry: antibodies are applied to thin ( 1-30 $\mu \mathrm{m})$ tissue sections and specifically bind to target antigens. Conjugated chromogens or fluorescent reporters are used to visualize the distribution of the target biomolecule via microscopy. Chromogens are typically used as cell-specific markers, while fluorophores are better suited to sub-cellular imaging.

Inductively coupled plasma-mass spectrometry (ICP-MS): an isotope-specific detector with high sensitivity, wide linear dynamic range, and ability to detect multiple analytes in a single experiment. A high-temperature argon plasma (8-10,000 K) atomizes and ionizes samples that are detected by a mass spectrometer based on their mass-to-charge $(\mathrm{m} / \mathrm{z})$ ratio.

Imaging mass cytometry: a commercial solution for highly multiplexed imaging of tissue samples using proprietary polymer linkers to pre-label primary antibodies with isotopically enriched lanthanide ions. A high-resolution LA system coupled to a heavily modified ICP-MS with a simultaneous time-of-flight mass analyzer can theoretically measure up to 100 individual analytes in a single imaging experiment.

Laser ablation (LA): focused UV lasers are directed onto the surface of a solid sample, vaporizing material that is swept by a carrier gas to an ICP-MS. The ICP-MS detects analytes in individual lines of ablation as a time-resolved signal, which are then reconstructed as twodimensional images. 
Metal chelating polymers (MCPs): soft materials that bind inorganic metal centers and nanostructures and link with organic macromolecules including antibodies [13].

Multiplexing: multiple antibodies specific to individual antigens are applied to a tissue sample to allow visualization of multiple target biomolecules in a single experiment.

Skewing: streaks or a signal gradient being observed in an image, potentially underrepresenting or overestimating the concentration of the analytes.

Upconversion nanoparticles (UCNPs): lanthanide-doped nanoparticles that emit photons in the UV range when excited by near-IR light [14]. Tuning phsyical properties such as particle diameter allows precise control of the number of lanthanide atoms per particle. 

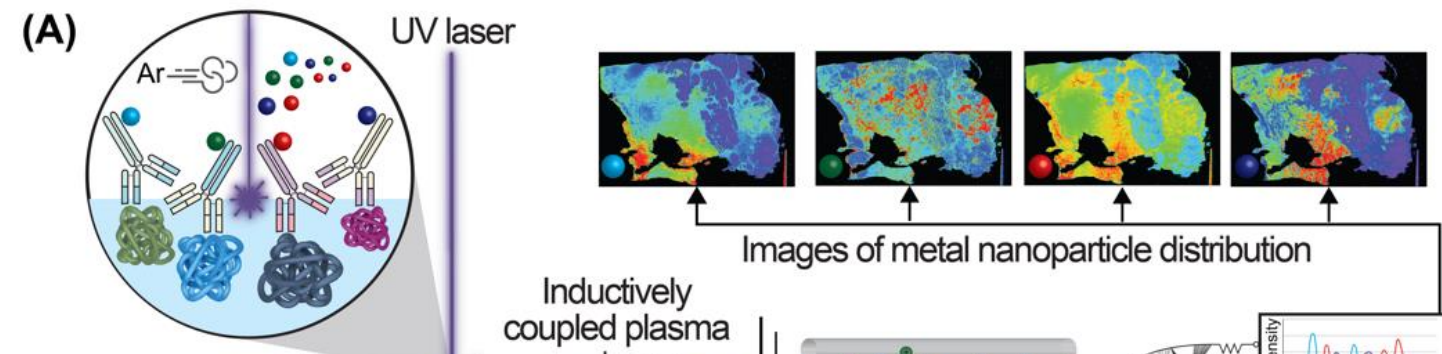

Inductively

Images of metal nanoparticle distribution coupled plasma

Target
antigens

O Metal

O conjugates

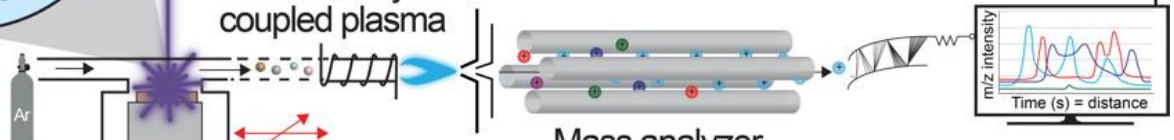

(B)
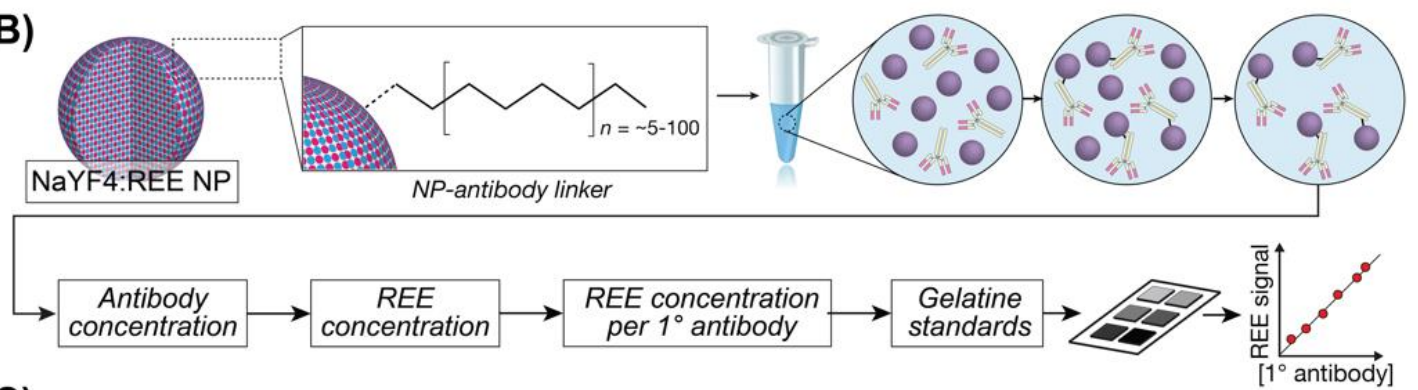

(C)

(D)
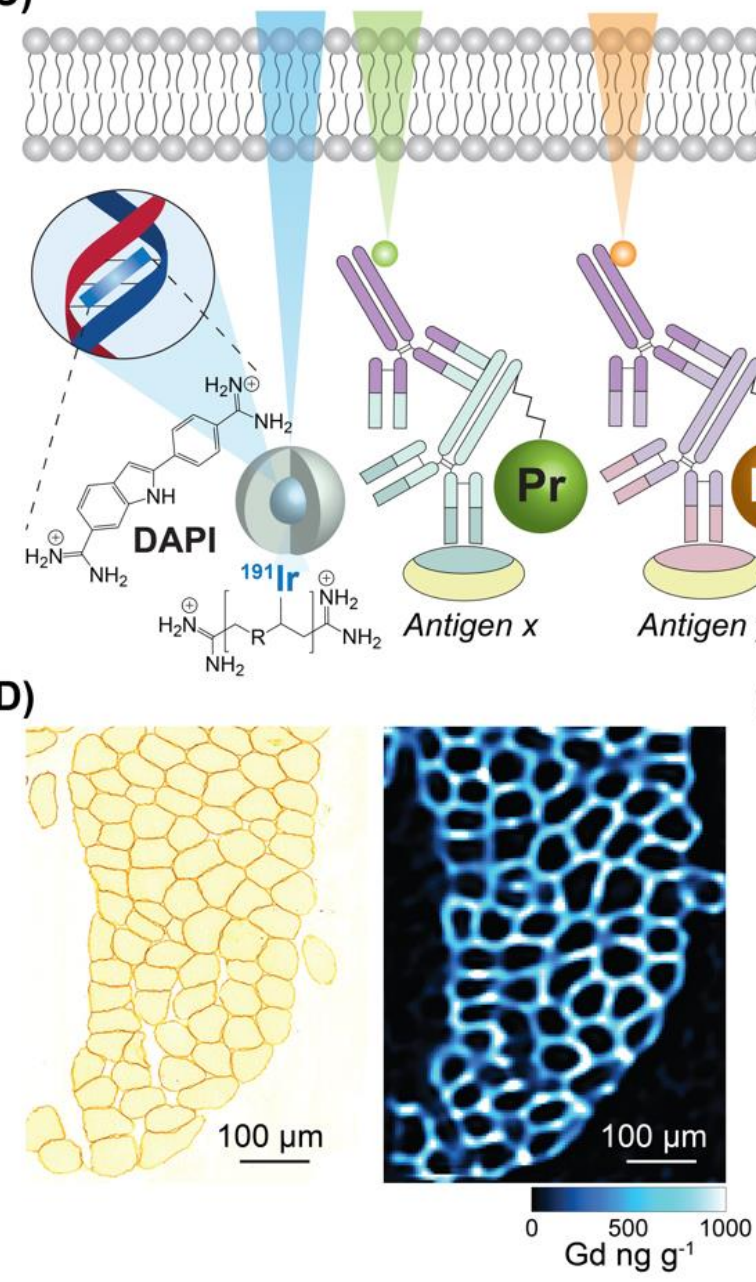

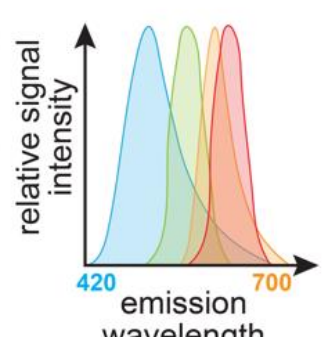

wavelength

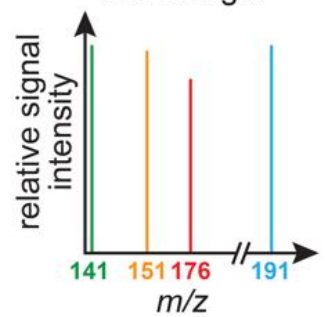

(E)

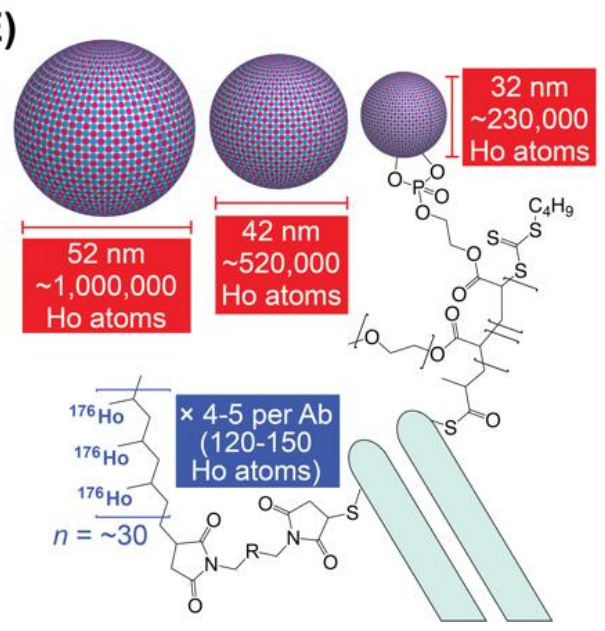


Figure 1. Rare earth elements as chemical reporters for iMSI. (A) Schematic of iMSI analysis workflow. Samples are immunolabelled with NP-conjugated antibodies (Abs) for multiple target antigens following standard protocols. Sections are then imaged by scanning the surface with a 1-100 $\mu \mathrm{m}$ laser beam and detecting isotopes of metals unique to each NP using ICP-MS. The mass-to-charge ratio $(\mathrm{m} / \mathrm{z})$ signal for each isotope is then used to reconstruct images in two or three dimensions. (B) Schematic for UCNP-labelling of $1^{\circ}$ Abs. A linking polymer is attached to the surface of the NP, it is incubated in excess with the monoclonal $1^{\circ}$ $\mathrm{Ab}$, and removed with a molecular weight filter. Ab and NP metal concentration are used to determine atoms per Ab for external calibration. (C) Higher levels of multiplexing is available with iMSI as it is not affected by spectral overlap. (D) Validation and benchmarking of an antibody requires a corresponding IHC image (left panel) to iMSI (right panel) on a control section to ensure the antibody is working as expected. (E) Lanthanide atoms per $1^{\circ} \mathrm{Ab}$ using Maxpar $^{\circledR}$ and UCNPs with variable diameters. 


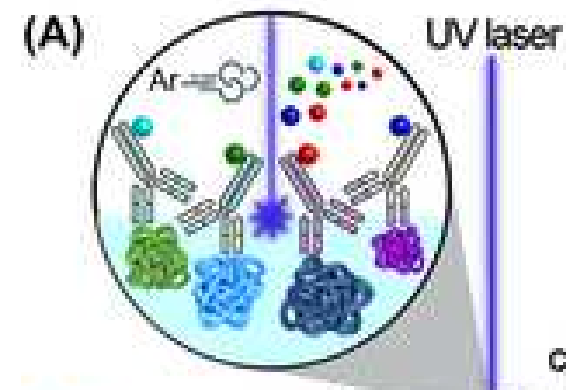

Inductively
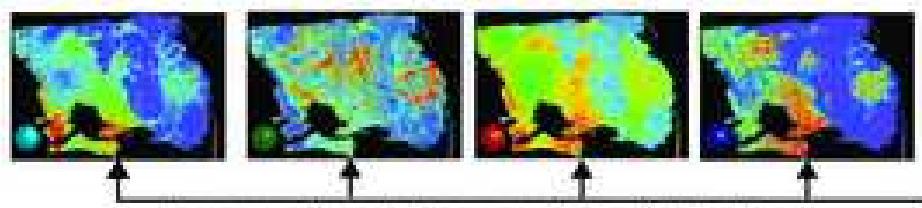

Images of metal nanoparticle distribution coupled plasma

(5) Target

弱悸 antigens

O Metal

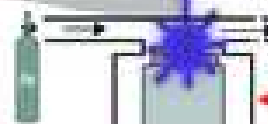

- conjugates: Laser ablation

(B)
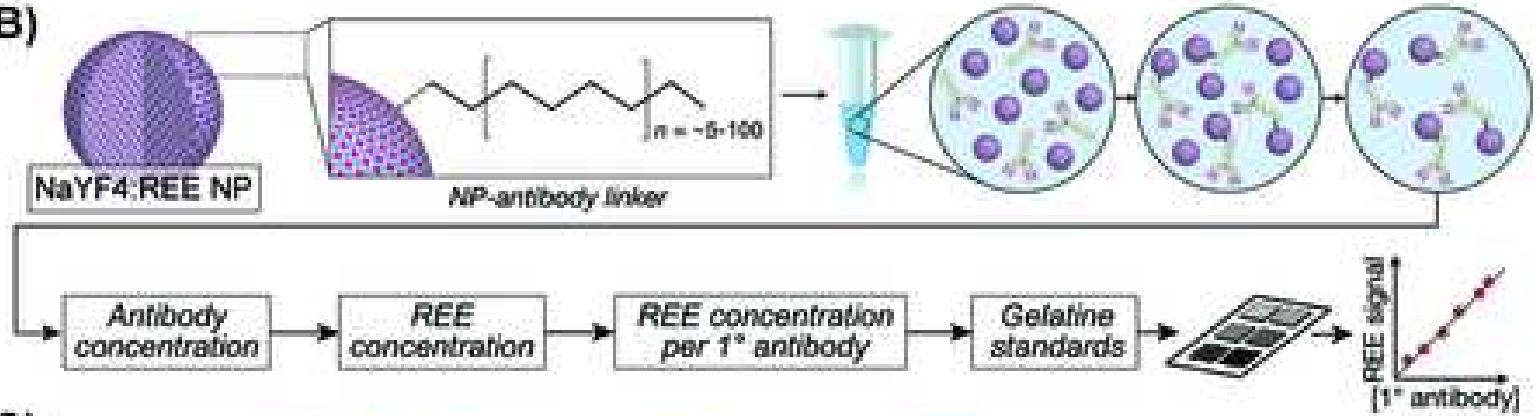

(C)

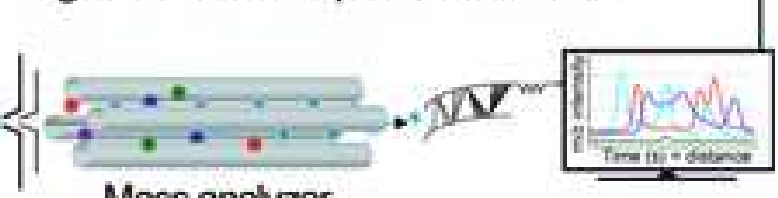

Mass analyzer
(D)
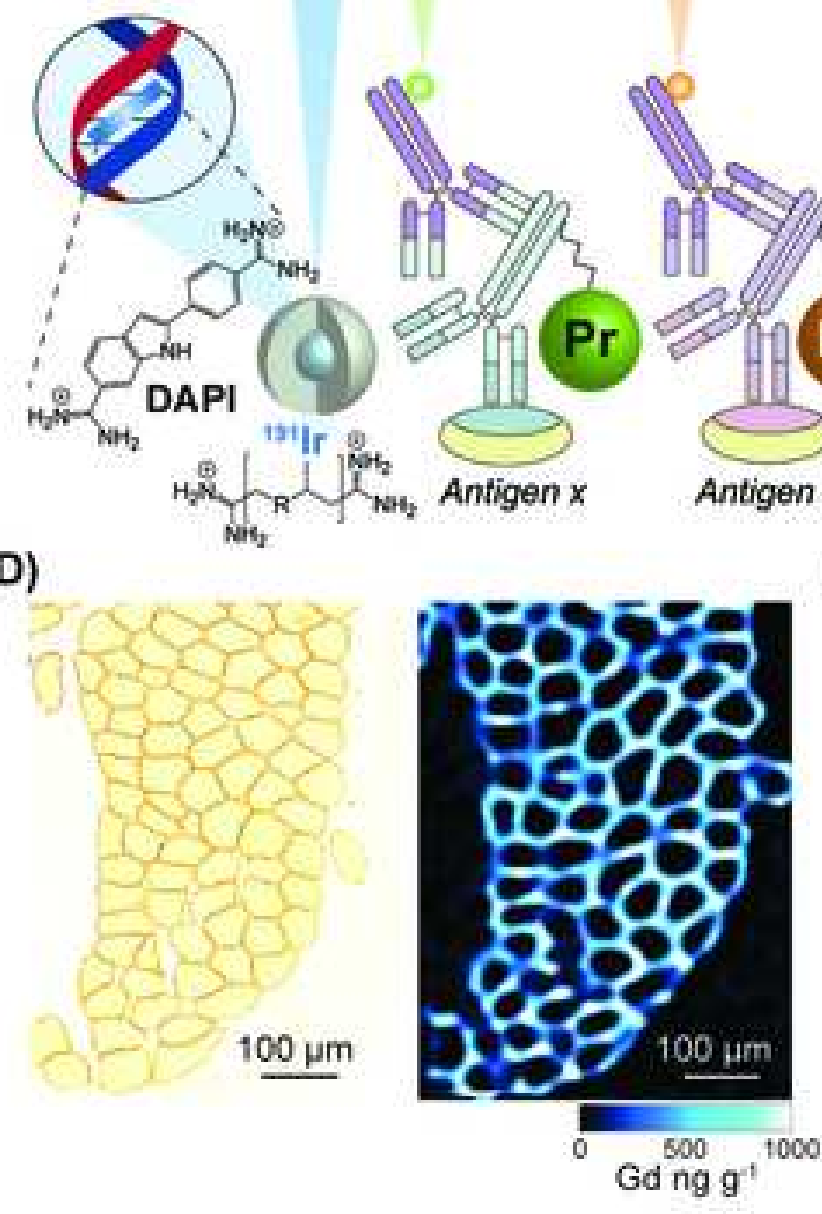

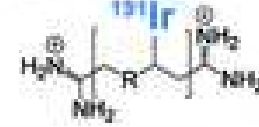

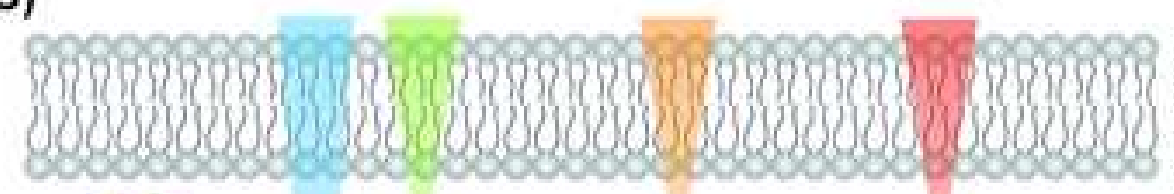

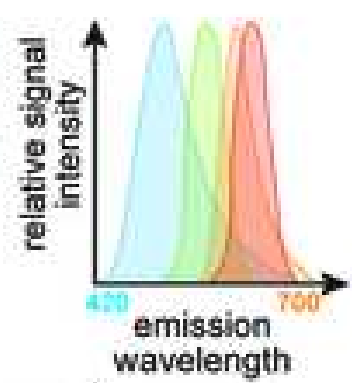

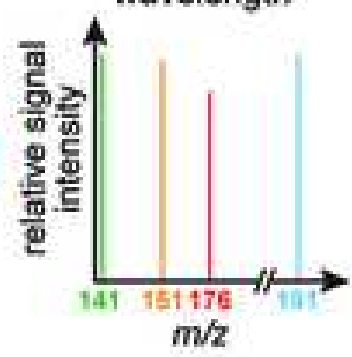

(E)

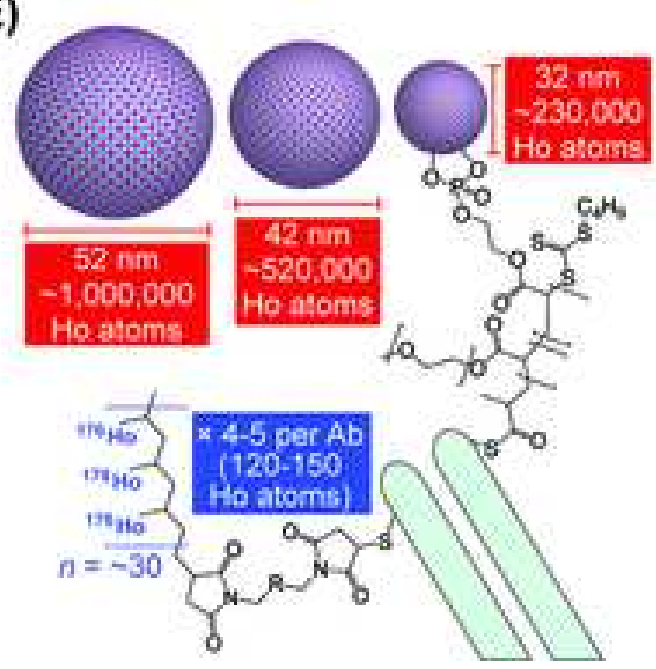


